\title{
A comprehensive study of Cepheid variables in the Andromeda galaxy ${ }^{\star}$
}

\section{Period distribution, blending, and distance determination}

\author{
F. Vilardell ${ }^{1}$, C. Jordi ${ }^{1,3}$, and I. Ribas ${ }^{2,3}$ \\ 1 Departament d'Astronomia i Meteorologia, Universitat de Barcelona, c/ Martí i Franquès, 1, 08028 Barcelona, Spain \\ e-mail: [francesc.vilardell; carme.jordi]@am.ub.es \\ 2 Institut de Ciències de 1'Espai-CSIC, Campus UAB, Facultat de Ciències, Torre C5-parell-2a, 08193 Bellaterra, Spain \\ e-mail: iribas@ieec.uab.es \\ 3 Institut d'Estudis Espacials de Catalunya (IEEC), Edif. Nexus, c/ Gran Capità, 2-4, 08034 Barcelona, Spain
}

Received 28 May 2007 / Accepted 18 July 2007

\begin{abstract}
Extragalactic Cepheids are the basic rungs of the cosmic distance scale. They are excellent standard candles, although their luminosities and corresponding distance estimates can be affected by the particular properties of the host galaxy. Therefore, the accurate analysis of the Cepheid population in other galaxies, and notably in the Andromeda galaxy (M31), is crucial to obtaining reliable distance determinations. We obtained accurate photometry (in $B$ and $V$ passbands) of 416 Cepheids in M 31 over a five year campaign within a survey aimed at the detection of eclipsing binaries. The resulting Cepheid sample is the most complete in M 31 and has almost the same period distribution as the David Dunlap Observatory sample in the Milky Way. The large number of epochs ( 250 per filter) has permitted the characterisation of the pulsation modes of 356 Cepheids, with 281 of them pulsating in the fundamental mode and 75 in the first overtone. The period-luminosity relationship of the fundamental mode Cepheids has been studied and a new approach has been used to estimate the effect of blending. We find that the blending contribution is as important as the metallicity correction when computing Cepheid distance determinations to M31 ( 0.1 mag). Since large amplitude Cepheids are less affected by blending, we have used those with an amplitude $\mathcal{A}_{V}>0.8 \mathrm{mag}$ to derive a distance to $\mathrm{M} 31$ of $(m-M)_{0}=24.32 \pm 0.12 \mathrm{mag}$.
\end{abstract}

Key words. Stars: variables: Cepheids - stars: distances - galaxies: individual: M 31 - galaxies: distances and redshifts methods: observational

\section{Introduction}

Cepheids are probably the most studied variable stars. Their large amplitudes and intrinsic luminosities make them easily detectable in most photometric variability surveys. In addition, their well-known period-luminosity (P-L) relationship (Leavitt \& Pickering 1912) has made these variable stars one of the main cornerstones in deriving extragalactic distances (see Freedman et al. 2001, for an historical review). The importance of Cepheids for distance determination stands in contrast with the relative lack of additional information on the specific characteristics of extragalactic Cepheids and the possible corrections because of their particular properties (i.e., metallicity). A clear example is the Andromeda galaxy (M31), where the first identification of Cepheids was already performed by Hubble (1929). After the observations of Baade \& Swope (1965, and references therein), few efforts have been dedicated to further analyze the Cepheid population in M 31.

This trend has changed in recent years with the emergence of new observational capabilities. Several variability surveys have started to study the stellar content in M31 (Macri 2004, and references therein) and other Local Group galaxies

* Based on observations made with the Isaac Newton Telescope operated on the island of La Palma by the Isaac Newton Group in the Spanish Observatorio del Roque de los Muchachos of the Instituto de Astrofísica de Canarias.
(Udalski et al. 1999; Mochejska et al. 2001; Udalski et al. 2001; Pietrzyński et al. 2004; Macri et al. 2006), obtaining large samples of Cepheids with accurate photometry. The detailed study of the observed Cepheids has emphasized the importance of an issue that was usually overlooked in most photometric studies: the effect of blending. It has been proposed (Mochejska et al. 2000, hereafter MMSS00) that the magnitude of Cepheids may be affected by the light of unresolved companion stars (i.e., blends). The effect of blending is somewhat different from crowding or confusion noise, since companion stars appear to be in the same point-like source. Therefore, even when achieving a perfect point-spread function modeling, blending could still be present. The effect can be the same as in spectroscopic binaries, where the individual components cannot usually be resolved from ground-based images.

When studying extragalactic Cepheids the spatial resolution decreases linearly with the distance of the host galaxy and, as a consequence, the number of possible blends increases. Small blending values are expected for the Large Magellanic Cloud (LMC), where individual Cepheids can be resolved from neighboring stars, even from ground-based observations. The situation changes in M31 and M33, where mean blending values of $0.18 \mathrm{mag}$ and $0.16 \mathrm{mag}$, respectively, have been obtained (Mochejska et al. 2004, hereafter MMSS04). These results would imply, when extrapolated to more distant galaxies, a downward revision of the Hubble constant between $5 \%$ to 
$10 \%$ (as explained by Gibson et al. 2000). By contrast, Bresolin et al. (2005) found an upper limit on blending in NGC 300 (at $\sim 2 \mathrm{Mpc}$ ) of $0.04 \mathrm{mag}$. In addition, Gibson et al. (2000) showed that the systematic effect of blending on farther galaxies (between 4 and $25 \mathrm{Mpc}$ ) is almost negligible. Therefore, results obtained so far seem to indicate that blending has an important contribution when observing Local Group galaxies and diminishes when observing distant galaxies. Gibson et al. (2000) explained such behavior as a consequence of the background levels in M 31 and M 33 (with a large number of stars detected around Cepheid variables) not being representative of the more distant galaxies (where the background levels are the result of several unresolved sources). Because of the importance of the subject, a comprehensive study of the effect of blending on Cepheid distance determinations is highly desirable.

One of the most recent variability surveys in M 31 was carried out by us and was centered on the North-Eastern quadrant of the galaxy (Vilardell et al. 2006, hereafter Paper I). Our main goal was to detect eclipsing binaries, although a sample of 416 Cepheids also resulted from the reduction and analysis process. The large number of detected stars and the high quality of the resulting light curves have allowed the current study of Cepheid properties in M 31 .

\section{Photometric sample}

The photometric survey in Paper I was carried out with the 2.5 m Isaac Newton Telescope (INT) at La Palma (Spain) over the course of five observing runs (between 1999 and 2003). An implementation of the difference image analysis technique (Wozniak 2000) was used in the reduction process to automatically detect 3964 variable stars with $\sim 250$ observations per band $(B$ and $V$ ). The analysis of variance algorithm (SchwarzenbergCzerny 1996) was used to identify periodic variable stars. The folded light curves were visually inspected, which led to the identification of 437 eclipsing binaries and 416 Cepheids. The photometric measurements were transformed to the standard system through the observation of several Landolt (1992) star fields, obtaining magnitude errors between 0.03 and $0.11 \mathrm{mag}$. Finally, the phase-weighted mean magnitudes (Saha $\&$ Hoessel 1990) of the Cepheid sample were determined for each passband ( $B$ and $V$ ). Figure 1 shows the color-magnitude diagram of the observed stars with good photometric precision. Cepheid variables are highlighted.

\section{Period distribution}

The period distribution of Cepheids in Local Group galaxies has been a major source of debate. Evolution models predict a displacement on the peak of the period distribution as a function of the metallicity of the host galaxy (Becker et al. 1977). It has also been observed that the Milky Way (MW) period distribution displays a dip at around 10 days, while such a feature is missing in more metal-poor galaxies such as the LMC. Several theories have been put forward to explain the bimodal distribution, but a satisfying scenario is still lacking (see Antonello et al. 2002, for an extended discussion).

Since the MW and M 31 have similar morphological type and chemical composition, the observed period distributions are also expected to be similar. The results obtained so far have prevented a direct comparison because of observational biases, which are often difficult to evaluate. On the one hand, faint Cepheids (with usually short periods) can be missing in some

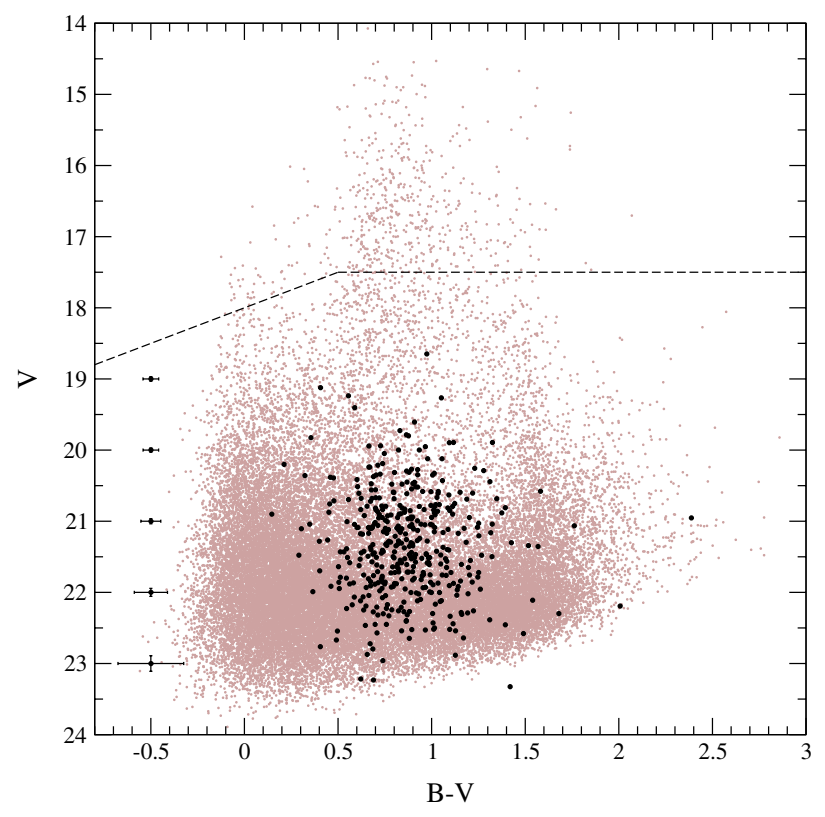

Fig. 1. Color-magnitude diagram for 37241 objects with photometric errors below $0.1 \mathrm{mag}$ in the reference catalog of Paper I (grey dots) and the 416 identified Cepheids (black circles). Objects above the dashed line are saturated. Error bars on the left indicate the mean error, in magnitude and color, at different magnitude values.

shallow surveys while, on the other hand, the distribution of the observations can make the identification of long period Cepheids difficult.

To evaluate the observational biases, the 416 Cepheids from Paper I were compared with the 420 Cepheids in M 31 of the GCVS (Samus et al. 2004). Both samples have almost the same number of stars, but the two period distributions (Fig. 2) are found to be largely different, as demonstrated by the Kolmogorov-Smirnov tests, which provide values of around $10^{-13}$. The origin of the observed difference can be explained by two main factors. Firstly, the Paper I sample is known to have a bias for the longest period Cepheids because of the observational window function (see Paper I for further information). And secondly, the GCVS presents an important bias at short periods because the GCVS is shallower than our survey and faint Cepheids are missing. Furthermore, both Cepheid samples in M 31 can be compared with the MW sample of the David Dunlap Observatory $^{1}$ (DDO, Fernie et al. 1995). As can be seen (Fig. 2), the period distributions of Paper I and the DDO sample are very similar (with a Kolmogorov-Smirnov test value of 0.49 ). Only a slight difference is observed at long periods, possibly because of the observational bias in the Paper I sample. Therefore, as expected, equivalent period distributions are obtained for similar galaxies, including the secondary peak at $P>10 \mathrm{~d}$ in metal rich galaxies.

The conspicuous similarity between the period distributions of MW and M31 becomes even more striking when compared with a galaxy of different metallicity (Fig. 2). More than 1300 Cepheids have been observed in the LMC as part of the OGLE II survey $^{2}$ (Udalski et al. 1999). The resulting period distribution reveals a single peak, which, as expected, is shifted towards shorter periods with respect to those of the distributions

\footnotetext{
1 Data obtained from: http://www . astro.utoronto.ca/DD0/ research/cepheids/cepheids.html

2 Data obtained from: ftp://sirius.astrouw.edu.pl/ogle/ ogle2/var_stars/lmc/cep/catalog/
} 


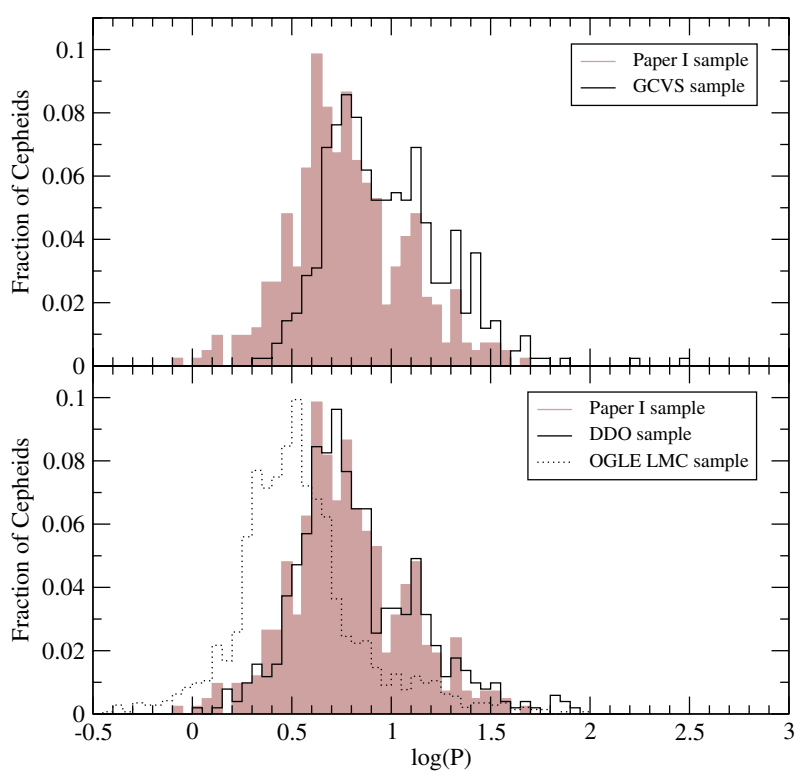

Fig. 2. Top: normalized period distribution for the 416 detected Cepheids in Paper I compared with the 420 Cepheids in M 31 of the GCVS. Bottom: normalized period distribution for the 416 detected Cepheids in M 31 compared with the 509 Cepheids in the MW from the DDO sample. The OGLE LMC Cepheid sample is also shown for comparison.

of the MW and M 31. Therefore, the great similarity between the DDO and Paper I samples indicates that both the observational biases and period distributions are equivalent.

\section{Fourier decomposition}

It is well known that Fourier decomposition of the Cepheid light curves can provide valuable information on the real nature of these variable stars, especially on their pulsation modes (Beaulieu et al. 1995). The procedure involves fitting the coefficients $\left(A_{k}\right.$ and $\left.\varphi_{k}\right)$ of a Fourier series of the form:

$m(t)=A_{0}+\sum_{k=1}^{J} A_{k} \cos \left(2 \pi k \Phi(t)+\varphi_{k}\right)$

where $m(t)$ and $\Phi(t)$ are the magnitude of the Cepheid and the phase at time $t$, respectively. Once the best coefficients have been obtained, the pulsation mode of the Cepheid variable can be derived from the amplitude ratio $R_{k 1}=A_{k} / A_{1}$ and phase difference $\varphi_{k 1}=\varphi_{k}-k \varphi_{1}$.

A prerequisite of the Fourier decomposition of the 416 Cepheids identified in M 31 is the determination of the maximum order of the fit, $J$. In agreement with other studies in the literature (Antonello et al. 1990; Beaulieu et al. 1995; Zakrzewski et al. 2000), we observed that an iterative solution provided satisfactory results. Therefore, for each light curve, the fitting procedure started with $J=1$ and increased until the value of $\chi_{\text {d.o.f. }}^{2}$ varied by less than one. Although the Fourier fit always provided values for all the light curves, several important aspects were considered before studying the results. Firstly, since measurements with large photometric errors can provide unreliable fits, light curves with a mean photometric error larger than $0.1 \mathrm{mag}$ were excluded. Secondly, all light curves with $\chi_{\text {d.o.f. }}^{2}>7$ were rejected to eliminate the fits that did not accurately match the observations. Finally, large gaps in a light curve can prevent
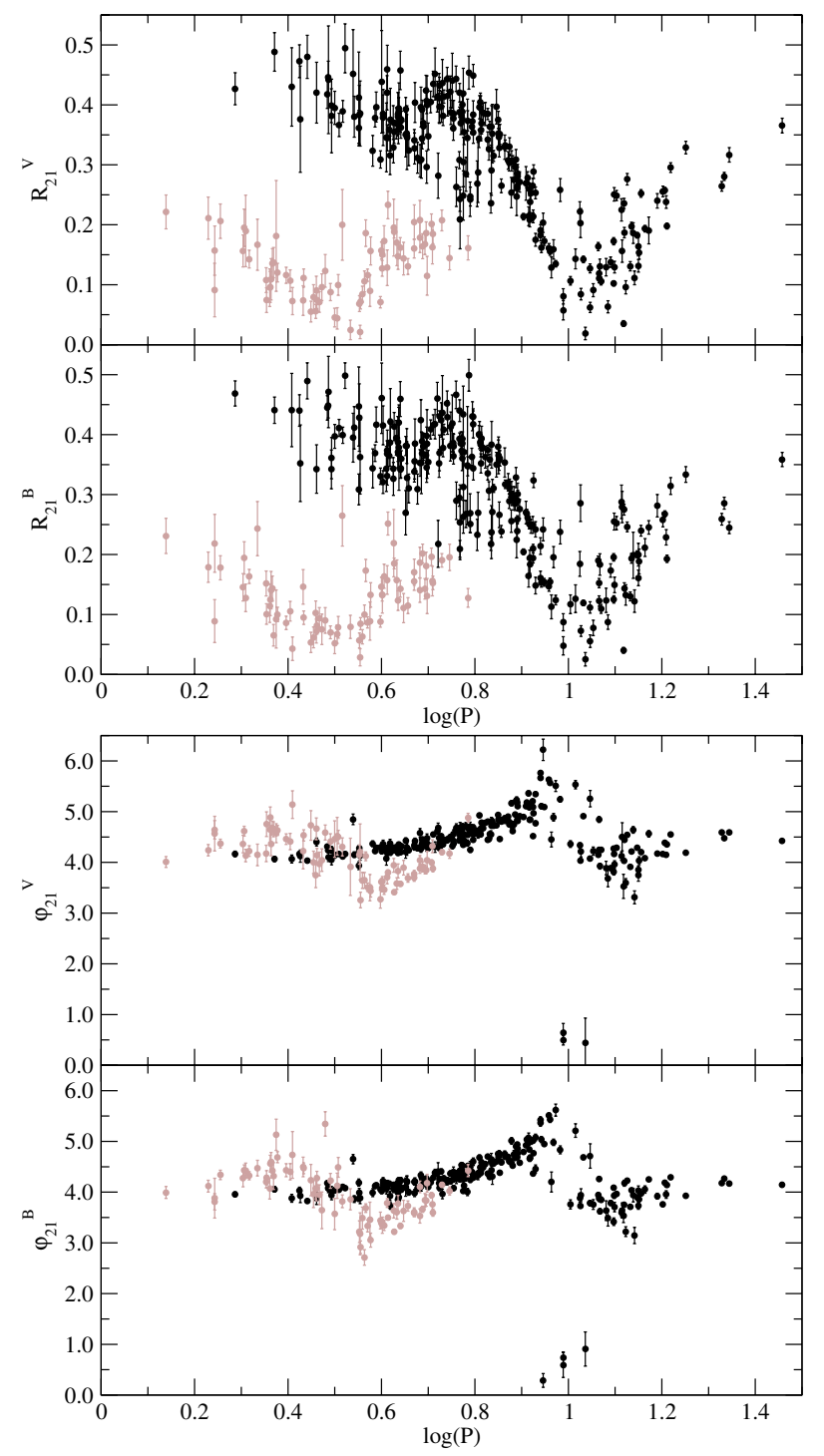

Fig. 3. Fourier coefficients in each passband $(B$ and $V)$ as a function of period. The pulsation mode of the 315 Cepheids is identified: black circles - fundamental mode; grey circles - first overtone.

the fit to correctly reproduce its shape, even when $\chi_{\text {d.o.f. }}^{2}$ is relatively low. Problematic fits usually have coefficients with large error bars. Therefore, coefficient errors were computed (according to Petersen 1986) and light curves with $\sigma_{A_{0}}>0.01 \mathrm{mag}$, $\sigma_{R_{21}}>0.1$, or $\sigma_{\varphi_{21}}>0.6 \mathrm{rad}(\simeq 0.2 \pi \mathrm{rad})$ were also rejected.

The above criteria yielded a sample of 315 Cepheids with accurate Fourier fits in both passbands ( $B$ and $V$ ). The $R_{21}$ and $\varphi_{21}$ values in both passbands (Fig. 3) have been used to classify 75 Cepheids pulsating in the first-overtone mode (FO) and 240 pulsating in the fundamental mode (FM). Since FO Cepheids are not expected to have periods longer than $\sim 7$ days, the FM sample has been completed with all Cepheids with $\log P>0.9(\simeq 8$ days $)$, regardless of the quality of their Fourier fit. The final sample of 281 FM Cepheids includes three type II Cepheid candidates, but they are easily identified as a consequence of the detailed analysis of the P-L distribution (Sect. 5.1). 


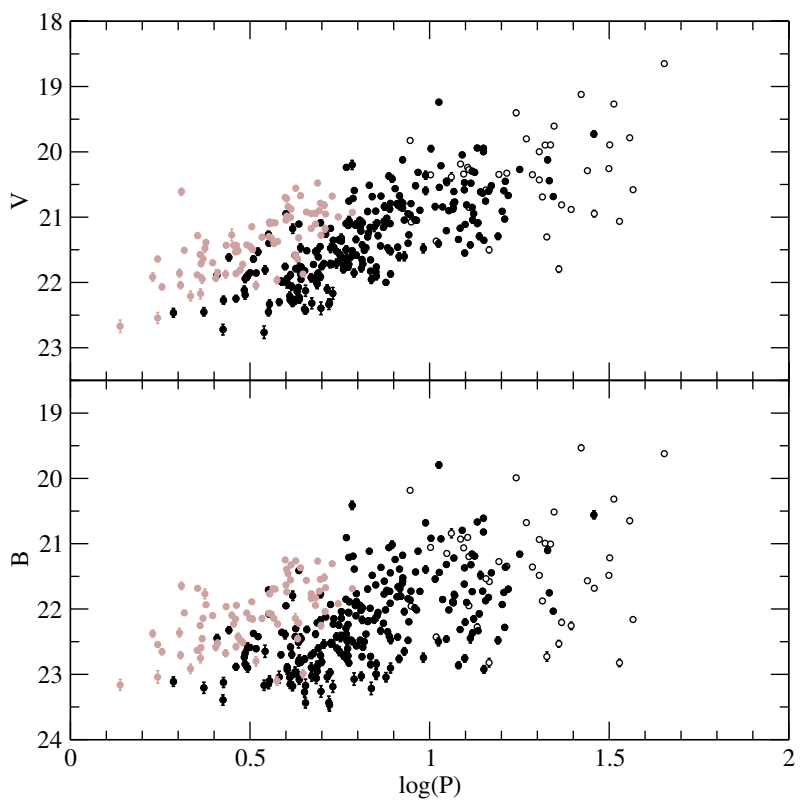

Fig. 4. Observed magnitudes as a function of period for 356 Cepheids. Filled black circles: fundamental mode Cepheids with accurate Fourier coefficients. Empty black circles: fundamental mode Cepheids without accurate Fourier coefficients. Grey circles: first overtone mode pulsators.

\section{Period-luminosity relationship}

The P-L diagram for the 356 classified Cepheids (281 FM and 75 FO) reveals a large scatter in both passbands (Fig. 4), especially in $B$. The origin of the observed scatter can be understood if we consider that the field of view covers about $7 \mathrm{kpc}$ at the distance of M $31\left((m-M)_{0}=24.44 \pm 0.12 \mathrm{mag}\right.$, Ribas et al. 2005). The large covered area in M31, the fact that Cepheids are located along the spiral arms, and the clumpy structure of the interstellar medium (clearly observed in the survey images), indicates that differential absorption in the disk is most likely responsible for an important fraction of the observed scatter. In addition, the larger dispersion of the P-L distribution in $B$ compared to $V$ reinforces the interstellar absorption hypothesis.

Another possible effect on the observed scatter is metallicity. Several authors have suggested (see, i.e., Gieren et al. 2005) that the slope of the Cepheid P-L relationship is basically independent of metallicity. However, comparison of the tip of the red giant branch and Cepheid distance determinations to several galaxies has shown that a slight dependence on metallicity is present (Sakai et al. 2004). Therefore, a metallicity dependence on the zero point of the Cepheid P-L relationship could exist. Since metallicity within M31 decreases as a function of the galactocentric distance (Zaritsky et al. 1994), some of the observed scatter could also be introduced by metallicity differences.

In addition, the Cepheid measured photometry can be affected by blends. The mean seeing of our images is around 1 arcsec. Therefore, each observed point-like source corresponds to $\sim 4 \mathrm{pc}$ at the distance of M 31. Since Cepheids are usually located in young star clusters and associations, it is likely that the observed magnitude of a Cepheid can be the combination of several unresolved sources.

Below we analyse the three aforementioned effects that are the probable sources of the scatter in the P-L diagram.

\subsection{Absorption}

The effect of differential absorption can be partially corrected from the observed Cepheid color $(B-V)$ through the colorexcess $\left(E(B-V) \equiv(B-V)-(B-V)_{0}\right.$, Feast 1999). We used P-L relationships from Udalski et al. $(1999)^{3}$ to estimate $B_{0}$ and $V_{0}$ values. The LMC distance modulus was assumed to be $(m-M)_{0}=18.42 \pm 0.06 \mathrm{mag}$ and was computed from the weighted mean of all the LMC distance determinations with eclipsing binaries (Fitzpatrick et al. 2003). The reason for using LMC relationships (instead of those for the MW), was motivated by several recent results. First, Gieren et al. (2005) suggested that the MW Cepheid distances, mostly obtained through the Baade-Wesselink method, could be affected by a systematic bias when converting the observed radial velocities into pulsation velocities. Second, Macri et al. (2006) found that LMC P-L slopes provide a better agreement with the P-L distribution of Cepheids in NGC 4258 than MW relationships. Finally, the new parallax measurements of several MW Cepheids (Benedict et al. 2007; van Leeuwen et al. 2007) yield P-L slopes that seem to be in better agreement with the LMC than with previous MW relationships.

After obtaining the color excess for each Cepheid, a total-toselective extinction ratio of $\mathcal{R}_{V} \equiv A(V) / E(B-V)=3.1 \pm 0.3$ (Fitzpatrick 1999) was used to compute the absorption and the $V_{0}$ magnitude for every Cepheid. The resulting P-L distribution can be compared with the LMC P-L relationship (Fig. 5), assuming a M 31 distance modulus of $(m-M)_{0}=24.44 \pm 0.12 \mathrm{mag}$ (Ribas et al. 2005). An offset between the $V_{0}$ values and the LMC $\mathrm{P}-\mathrm{L}$ relationship is clearly observed, but the effects of metallicity and blending have still to be considered.

Finally, as previously mentioned, three of the studied Cepheids seem to be of type II. To further investigate these objects they have been kept in the studied sample of FM Cepheids (Sects. 5.3 and 6).

\subsection{Metallicity}

As explained above, the zero point of the P-L relationship may depend on metallicity. To account for this effect, the metallicity of each Cepheid needs to be estimated, and we did so by considering a galactocentric metallicity dependence. According to Zaritsky et al. (1994), the M 31 galactocentric metallicity dependence can be modeled by:

$$
12+\log (\mathrm{O} / \mathrm{H})=(9.03 \pm 0.09)-(0.28 \pm 0.10)\left(\rho / \rho_{0}-0.4\right)
$$

where $\rho$ is the de-projected galactocentric radius and $\rho_{0}$ is the isophotal radius (77.44 arcmin for M 31). Following the procedure in Baade \& Arp (1964), the Cepheid galactocentric radius was obtained assuming a position angle of $38^{\circ}$, an inclination of 12.5 (Simien et al. 1978), and an M31 center position of $\alpha=00^{\mathrm{h}} 42^{\mathrm{m}} 44^{\mathrm{s}} .31 \delta=+41^{\circ} 16^{\prime} 09^{\prime} .4$ (in J2000.0 coordinates, Cotton et al. 1999).

The metallicity dependence of the Cepheid P-L relationship and the galactocentric metallicity variation implies that a different P-L relationship must be used for each individual Cepheid when computing the value $V_{0}-M_{V}$. Alternatively, one can compute $V_{0}-M_{V}$ by using a universal P-L relationship and then applying a correction for each Cepheid a posteriori. According to Sakai et al. (2004), the correction on the computed $V_{0}-M_{V}$

\footnotetext{
3 Updated relationships were obtained from the OGLE II web site: ftp://sirius.astrouw.edu.pl/ogle/ogle2/var_stars/lmc/ cep/catalog/README.PL
} 


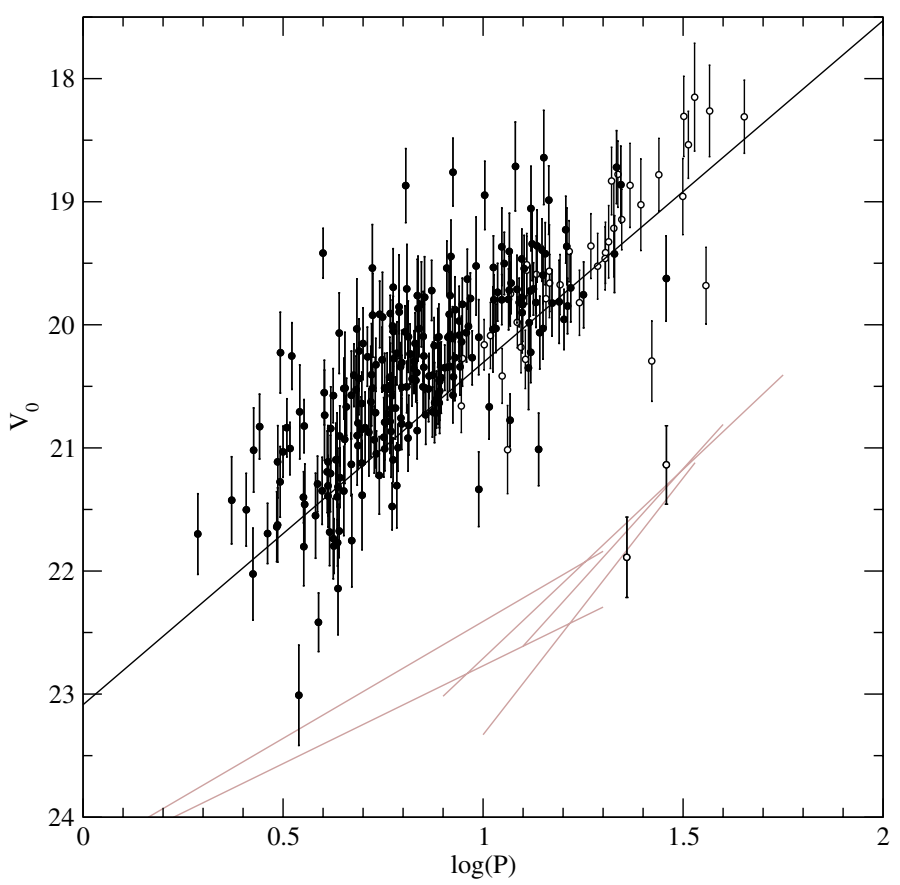

Fig. 5. Absorption-corrected $V$ magnitude as a function of period for 281 fundamental mode Cepheids. Filled circles: stars with accurate Fourier fit. Empty circles: stars without accurate Fourier fits. Black line: Udalski et al. (1999) P-L relationship, for a distance modulus to M31 of 24.44 mag (Ribas et al. 2005). Grey lines: distance corrected P-L relationships for type II Cepheids as given by Alcock et al. (1998).

value is $\delta(m-M)_{0} / \delta(\mathrm{O} / \mathrm{H})=-0.25 \pm 0.09 \mathrm{mag} / \mathrm{dex}$ when using the Udalski et al. (1999) P-L relationships. Therefore, we computed metallicity corrections by comparing the assumed metallicity of each M31 Cepheid with the metallicity of the LMC $(12+\log (\mathrm{O} / \mathrm{H})=8.5 \mathrm{dex}$, Sakai et al. 2004). The resulting corrections for the studied sample lie between -0.15 mag and -0.05 mag.

\subsection{Blending}

The best approach to study the effect of blending given our available data sets is by using the observed Cepheid amplitudes (Antonello 2002). The intrinsic amplitude $\mathcal{A}^{\mathrm{i}}$ of a Cepheid is given by the expression:

$\mathcal{A}^{\mathrm{i}}=-2.5 \log \left(\frac{f_{\mathrm{c}}}{F_{\mathrm{c}}}\right)$

where $f_{\mathrm{c}}$ and $F_{\mathrm{c}}$ are the fluxes at minimum and maximum light, respectively. When blending is present, the observed amplitude $\mathcal{A}$ can be expressed in the following form:

$\mathcal{A}=-2.5 \log \left(\frac{f_{\mathrm{c}}+f}{F_{\mathrm{c}}+f}\right)$

where $f$ is the sum of fluxes of all blending sources. From these two equations, one can write:

$\mathcal{A}=2.5 \log \left(\frac{10^{0.4 \mathcal{H}^{\mathrm{i}}}+\frac{f}{f_{\mathrm{c}}}}{1+\frac{f}{f_{\mathrm{c}}}}\right)$.

Since $\mathcal{A}^{\mathrm{i}}$ and $f / f_{\mathrm{c}}$ are always positive quantities, the observed amplitude of a blended Cepheid is always smaller than its intrinsic amplitude. The observed amplitude and the blended mean magnitude $\langle m\rangle$ can be expressed as:

$$
\begin{aligned}
\mathcal{A} & =m-M \\
\langle m\rangle & =\frac{m+M}{2}
\end{aligned}
$$

where $m$ and $M$ are the observed magnitudes at minimum and maximum light of a Cepheid, respectively. In the same way, the intrinsic amplitude and the blending-free mean magnitude $\left\langle m^{\mathrm{i}}\right\rangle$ can be expressed as:

$$
\begin{gathered}
\mathcal{A}^{\mathrm{i}}=m^{\mathrm{i}}-M^{\mathrm{i}} \\
\left\langle m^{\mathrm{i}}\right\rangle=\frac{m^{\mathrm{i}}+M^{\mathrm{i}}}{2} .
\end{gathered}
$$

From Eqs. (5)-(8) the difference on the mean magnitude of a given Cepheid as a consequence of blending can be expressed as:

$$
\Delta=\langle m\rangle-\left\langle m^{\mathrm{i}}\right\rangle=\left(m-m^{\mathrm{i}}\right)-\frac{\mathcal{A}-\mathcal{A}^{\mathrm{i}}}{2} .
$$

Considering that $\left(m-m^{\mathrm{i}}\right)$ can be defined as:

$$
m-m^{\mathrm{i}}=-2.5 \log \left(\frac{f_{\mathrm{c}}+f}{f_{\mathrm{c}}}\right)=-2.5 \log \left(1+\frac{f}{f_{\mathrm{c}}}\right)
$$

and isolating $f / f_{\mathrm{c}}$ from (4), the difference on the mean magnitude (9) can be expressed as:

$\Delta=2.5 \log \left(\frac{10^{0.2 \mathcal{A}}-10^{-0.2 \mathcal{A}}}{10^{0.2 \mathcal{A}^{\mathrm{i}}}-10^{-0.2 \mathcal{A}^{\mathrm{i}}}}\right)$.

Therefore, the variation on the mean magnitude of a given Cepheid because of blending can be computed from the amplitude of the Cepheid. Although $\mathcal{A}^{i}$ and $\Delta$ are unknown quantities, the above equation can be solved in combination with periodcolor and P-L relationships. The results shown below are based on the Udalski et al. (1999) relationships, but almost identical results were obtained with other LMC relationships (Sandage et al. 2004).

The next step of the process involves recalling that $\langle m\rangle \simeq \bar{m}$, where $\bar{m}$ is the phase-weighted intensity-average mean magnitude of the observed Cepheids. Therefore, defining the blendingfree color excess as:

$E\left(B^{\mathrm{i}}-V^{\mathrm{i}}\right) \equiv\left(B^{\mathrm{i}}-V^{\mathrm{i}}\right)-(B-V)_{0}$

the blended color excess can be expressed as:

$E(B-V)=E\left(B^{\mathrm{i}}-V^{\mathrm{i}}\right)+\Delta_{B}-\Delta_{V}$

where $\Delta_{B}=B-B^{\mathrm{i}}$ and $\Delta_{V}=V-V^{\mathrm{i}}$. Analogously, the distance modulus can be expressed as:

$\left(V_{0}-M_{V}\right)=(m-M)_{0}+\Delta_{V}-\mathcal{R}_{V}\left(\Delta_{B}-\Delta_{V}\right)$

where:

$$
\begin{gathered}
\left(V_{0}-M_{V}\right)=V-\mathcal{R}_{V} E(B-V)-M_{V} \\
(m-M)_{0}=V^{\mathrm{i}}-\mathcal{R}_{V} E\left(B^{\mathrm{i}}-V^{\mathrm{i}}\right)-M_{V} .
\end{gathered}
$$

The combination of Eqs. (14) and (11) reveals that the value of $\left(V_{0}-M_{V}\right)$ is only a function of the amplitude of the Cepheids and the distance modulus to M31. In addition, the fact that the color excess is obtained from the observed color of the Cepheids (which is affected by blending) introduces the color 


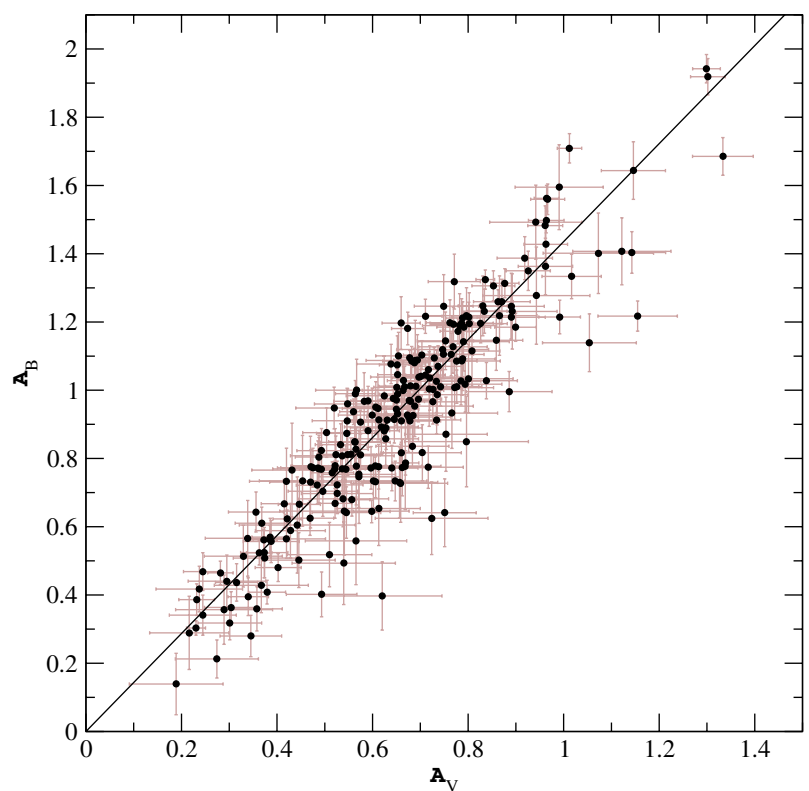

Fig. 6. Amplitude relationship for the 240 fundamental mode Cepheids.

term $\left(\Delta_{B}-\Delta_{V}\right)$ in Eq. (14). Therefore, and contrary to the intuitive interpretation, when reddening is computed from the observed color of Cepheids $(B-V)$ the blended distance modulus can be either larger or smaller than the intrinsic distance modulus $(m-M)_{0}$, depending on the values of $\left(\Delta_{B}-\Delta_{V}\right)$.

Considering that all Cepheids are roughly at the same distance, $(m-M)_{0}=24.44 \pm 0.12$ mag (Ribas et al. 2005), and assuming that $\mathcal{A}_{V}^{\mathrm{i}}$ and $\mathcal{A}_{B}^{\mathrm{i}}$ are linearly dependent (Fig. 6), Eq. (14) can be numerically solved. In fact, when the intrinsic amplitude of the Cepheid tends to zero, the observed amplitudes have to be zero in all passbands. Therefore, both amplitudes were considered to be proportional (i.e., $\mathcal{A}_{B}^{\mathrm{i}}=\alpha \mathcal{A}_{V}^{\mathrm{i}}$ ). The assumption that Cepheid amplitudes, when observed at different passbands, are proportional can be considered a first-order approximation of the Fourier coefficient interrelations (Ngeow et al. 2003).

Since the interrelations between $B$ and $V$ have not been accurately worked out, we computed the proportionality factor empirically from the observed amplitude of Cepheids. The amplitude of the studied Cepheids can be reliably estimated from the Fourier fits (Sect. 4). Therefore, the 240 FM Cepheids with accurate Fourier fits were used to compute the amplitude proportionality factor (Fig. 6), obtaining a mean value (with $2.5 \sigma$ clipping) of $\mathcal{A}_{B}^{\mathrm{i}} / \mathcal{A}_{V}^{\mathrm{i}} \simeq \mathcal{A}_{B} / \mathcal{A}_{V}=1.435 \pm 0.011$.

The above distance modulus to M 31 and the amplitude proportionality factor, when applied to Eq. (14), yielded the intrinsic amplitudes of the Cepheids. Once the intrinsic amplitudes are known, the difference on the mean magnitude can be computed (Fig. 7). The large resulting uncertainties in $\Delta_{B}$ and $\Delta_{V}$ clearly indicate that not much can be said from the individual blending of each Cepheid. The uncertainties were obtained from a Monte Carlo run with 1000 realizations on the input parameters of each Cepheid. The positive $\Delta$ values (implying negative blending) provide additional information on the associated uncertainties (probably of the order of 0.1-0.2 mag). In any case, it is interesting to observe that moderate intrinsic amplitudes are obtained for the entire sample, as it can be deduced from the lines of constant intrinsic amplitude in Fig. 7 (at $\Delta=0, \mathcal{A}=\mathcal{A}^{\mathrm{i}}$ ). Furthermore, the Cepheid with $\Delta_{V}>1 \mathrm{mag}$ is the only suspected type II Cepheid with an accurate Fourier fit (see Fig. 5). The unrealistic blending value provides an additional evidence in favor of the type II classification.
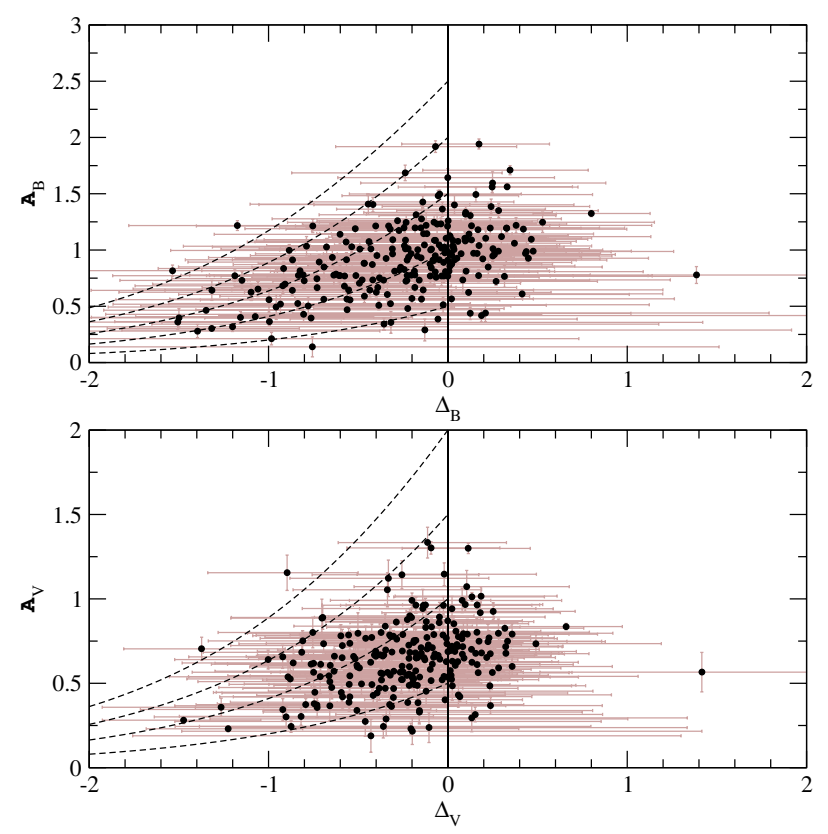

Fig. 7. Observed amplitude of the 240 fundamental mode Cepheids with accurate Fourier fits as a function of the computed variation on the mean magnitude due to blending. Lines of constant intrinsic amplitude from 0.5 to 2 or $2.5 \mathrm{mag}$ in steps of $0.5 \mathrm{mag}$ are also shown (dashed lines).

Given the large number of studied Cepheids, the obtained blending values can also be used to determine a mean blending value for Cepheids in M31. The results are shown in Table 1 where they are also compared with previous blending determinations in M31 and M33. The values in Paper I were obtained from the third light contribution in eclipsing binary systems, and the MMSS00 and MMSS04 values were obtained from comparison of Hubble Space Telescope (HST) and ground based images. The blending factors $(S)$ presented in MMSSO0 and MMSS04 were transformed into variation on the mean magnitude values by considering that:

$\Delta=-2.5 \log \left(1+\frac{f}{\left\langle f_{\mathrm{c}}\right\rangle}\right) \equiv-2.5 \log (1+S)$

where $\left\langle f_{c}\right\rangle$ is the flux of the Cepheid at mean magnitude.

The observed difference with MMSSO0 could be due to the assumed distance moduli or to the observing conditions. On the one hand, the reported uncertainties on the LMC and M 31 distance moduli could produce a variation of 0.12 mag in $\Delta_{B}$ and $\Delta_{V}$. On the other hand, blending depends on seeing conditions and background level. Large seeing images increase the blending and high signal-to-noise data enables the detection of faint stars, decreasing the computed background and increasing the blending contribution. Considering that similar results have been obtained for the eclipsing binary sample (which comes from the same observational data but from a completely different procedure), the obtained differences with the values reported in MMSS00 could be the result of different observing conditions. The method used by MMSSO0 can only provide lower limits to blending values, since the HST point spread function (used as a blending-free reference) could still hide unresolved companions. This is especially the case for the $B$-band results, where the available HST data was of low signal-to-noise, thus implying that only the most severe blends were detected. In addition, the relatively small sample used by MMSSO0 cannot be considered representative of the Cepheid population in M 31 . 
Table 1. Blending values in M 31 and M 33 obtained from several methods. Top: mean values and their errors. Bottom: median values.

\begin{tabular}{|c|c|c|c|c|c|c|}
\hline Reference & Sample & Number & $\left\langle S_{V}\right\rangle$ & $\left\langle\overline{\left\langle S_{B}\right\rangle}\right.$ & $\begin{array}{c}\left.\Delta_{V}\right\rangle \\
{[\mathrm{mag}]}\end{array}$ & $\begin{array}{c}\left\langle\Delta_{B}\right\rangle \\
{[\mathrm{mag}]}\end{array}$ \\
\hline MMSS00 & Cepheids in M31 & $22(10$ in $B)$ & $0.19 \pm 0.03$ & $0.06 \pm 0.06$ & $-0.18 \pm 0.03$ & $-0.05 \pm 0.05$ \\
\hline Paper I & Eclipsing binaries & 48 & $0.31 \pm 0.07$ & $0.30 \pm 0.06$ & $-0.23 \pm 0.05$ & $-0.25 \pm 0.04$ \\
\hline Present work & FM Cepheids & 240 & $0.31 \pm 0.03$ & $0.37 \pm 0.04$ & $-0.23 \pm 0.02$ & $-0.24 \pm 0.03$ \\
\hline Present work & FM Cepheids with $P>12$ days & 37 & $0.14 \pm 0.06$ & $0.20 \pm 0.10$ & $-0.09 \pm 0.05$ & $-0.10 \pm 0.07$ \\
\hline Present work & FM Cepheids with $\mathcal{A}_{V}>0.8 \mathrm{mag}$ & 37 & $0.10 \pm 0.05$ & $0.11 \pm 0.07$ & $-0.06 \pm 0.04$ & $-0.05 \pm 0.06$ \\
\hline MMSS04 & Cepheids in M 33 & $95(57$ in $B)$ & $0.24 \pm 0.03$ & $0.29 \pm 0.06$ & $-0.20 \pm 0.02$ & $-0.23 \pm 0.04$ \\
\hline MMSS04 & Cepheids in M 33 with $P>10$ days & $60(39$ in $B)$ & $0.16 \pm 0.04$ & $0.20 \pm 0.05$ & $-0.14 \pm 0.025$ & $-0.17 \pm 0.04$ \\
\hline Reference & Sample & Number & $\operatorname{Median}\left(S_{V}\right)$ & $\operatorname{Median}\left(S_{B}\right)$ & $\begin{array}{c}\operatorname{Median}\left(\Delta_{V}\right) \\
{[\mathrm{mag}]}\end{array}$ & $\begin{array}{c}\operatorname{Median}\left(\Delta_{B}\right) \\
{[\mathrm{mag}]}\end{array}$ \\
\hline MMSS00 & Cepheids & $22(10$ in $B)$ & 0.12 & 0.00 & -0.12 & 0.00 \\
\hline Paper I & Eclipsing binaries & 48 & 0.09 & 0.16 & -0.09 & -0.16 \\
\hline Present work & FM Cepheids & 240 & 0.20 & 0.15 & -0.19 & -0.16 \\
\hline Present work & FM Cepheids with $P>12$ days & 37 & 0.09 & 0.04 & -0.09 & -0.05 \\
\hline Present work & FM Cepheids with $\mathcal{A}_{V}>0.8 \mathrm{mag}$ & 37 & 0.09 & 0.04 & -0.09 & -0.05 \\
\hline MMSS04 & Cepheids in M 33 & $95(57$ in $B)$ & 0.13 & 0.15 & -0.13 & -0.15 \\
\hline MMSS04 & Cepheids in M 33 with $P>10$ days & $60(39$ in $B)$ & 0.07 & 0.10 & -0.07 & -0.10 \\
\hline
\end{tabular}

It is interesting to note that the results of MMSS04, based on a relatively large sample of Cepheids in M33, have mean values similar to ours (although slightly different median values; Table 1). Such good agreement should be taken with caution as the host galaxies, the methods used, the period distributions of the samples, and therefore the associated systematic errors are different. Nevertheless, it is encouraging that estimates made using different methods in two somewhat differing spiral galaxies yield similar results.

To further analyze the effects of blending we have applied several cuts to the FM sample. It has traditionally been argued that the longer period (and brighter) Cepheids should be less affected by blending (Macri et al. 2006). We computed the mean blending for Cepheids with a period longer than 12 days and obtained, as expected, lower blending values (Table 1). Furthermore, considering that blending decreases the Cepheid amplitude, larger amplitude Cepheids should also be less affected by blending and, in fact, the 37 Cepheids with $\mathcal{A}_{V}>$ 0.8 mag do have lower blending values (Table 1 ).

Finally, a blending-corrected color excess can be computed from Eq. (13), obtaining a mean value of $\left\langle E\left(B^{\mathrm{i}}-V^{\mathrm{i}}\right)\right\rangle=0.305 \pm$ 0.011 . The blended mean color excess is slightly lower $(\langle E(B-$ $V)\rangle=0.296 \pm 0.012)$, indicating that blending sources are bluer than Cepheids. The difference is more significant when considering only Cepheids with large blending values $\left(\Delta_{V}<-0.5 \mathrm{mag}\right.$ and $\Delta_{B}<-0.5 \mathrm{mag}$ ), obtaining a blending-corrected color excess of $\left\langle E\left(B^{\mathrm{i}}-V^{\mathrm{i}}\right)\right\rangle=0.472 \pm 0.028$, whereas $\langle E(B-V)\rangle=$ $0.315 \pm 0.030$. Therefore, considering the color-magnitude diagram (Fig. 1) and the distribution of Cepheids along the spiral arms, it is likely that early-type and young main sequence stars are responsible for the large measured blending. The obtained results are in good agreement with the results in MMSS04, who also found that M33 blending sources were on average bluer than the Cepheids. To further check this scenario we used young MW open clusters to compute the light contribution that the main sequence stars would introduce on a Cepheid variable in the cluster. The resulting predictions on $\Delta_{V}$ and $\Delta_{B}-\Delta_{V}$ are in good agreement with the inferred blending values in M 31 .

\section{Distance determination}

As previously mentioned, long period and large amplitude Cepheids are less affected by blends. Therefore, when computing the mean distance modulus to M31, a systematic trend should be observed for increasing period and amplitude cuts (i.e., rejecting short period or low amplitude Cepheids). The $V_{0}-$ $M_{V}$ values obtained after the metallicity correction (Sect. 5.2) were used to compute weighted mean distance determinations to M31 (with $2.5 \sigma$ clipping). The resulting values assume a distance modulus to the LMC of $(m-M)_{0}=18.42 \pm 0.06$ mag (Sect. 5.1). The amplitudes for the 41 FM Cepheids without accurate fits were computed from the observations and were also included in order to obtain a better coverage in periods and amplitudes.

Figure 8 shows that the distance modulus increases as the minimum period or minimum amplitude cuts increase. We observe that the distance modulus value stabilizes for $\mathcal{A}_{V}>0.8$ mag. The most likely cause for the observed tendency is that blending is lower than photometric errors (or even zero) for large amplitudes. The most suitable period cut is more difficult to compute, although an increasing tendency is also observed. The observed behavior is explained if long period Cepheids are still affected by large blends, thus still introducing a bias in the derived distance modulus. Hence, an amplitude cut is the best choice to derive the distance to M31 from the studied Cepheid sample. From this analysis (Fig. 8), the 66 Cepheids with amplitude $\mathcal{A}_{V}>0.8 \mathrm{mag}$ seem to represent the best sample for distance determination.

The effect of removing small amplitude Cepheids is clearly visible in Fig. 9, where the P-L diagram for the largest amplitude Cepheids is shown. Two stars with $\mathcal{A}_{V}>0.8$ mag are placed far below the general P-L distribution (empty circles in Fig. 9). Both stars are on the type II Cepheids relationships, reinforcing the hypothesis that these stars are, in fact, type II Cepheids and they have not been considered from now on. Finally, the obtained P-L slope, with a value of $-2.83 \pm 0.12 \mathrm{mag} \mathrm{dex}^{-1}$, is closer to the Udalski et al. (1999) LMC value of $-2.779 \pm 0.031 \mathrm{mag} \mathrm{dex}^{-1}$ than to MW P-L slopes (e.g.: $-3.087 \pm 0.085 \mathrm{mag} \mathrm{dex}^{-1}$, Sandage et al. 2004), therefore favoring the adopted P-L relationships. 


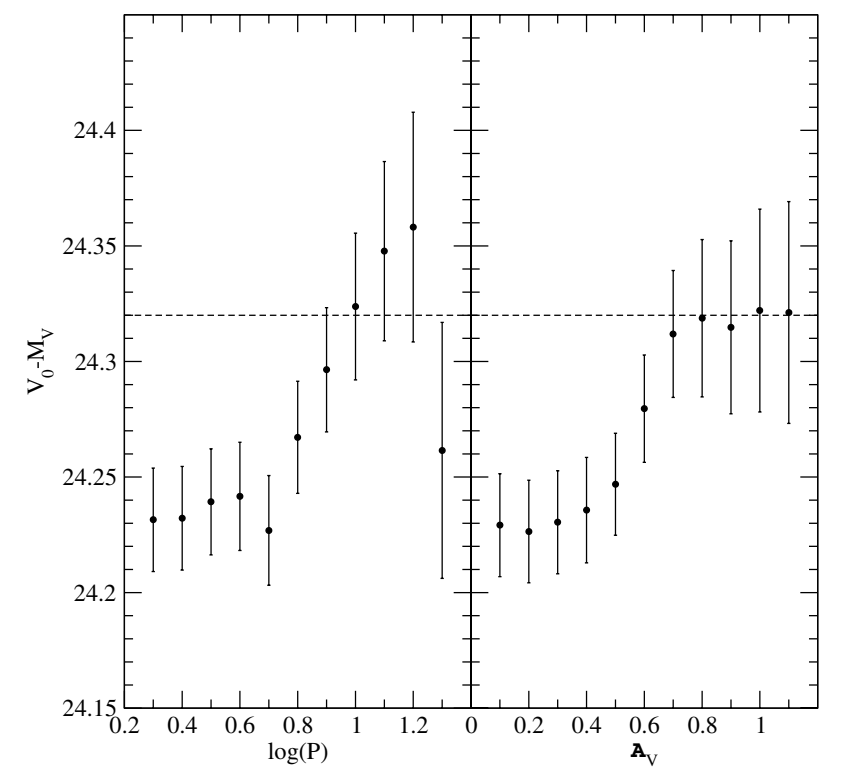

Fig. 8. Weighted mean $V_{0}-M_{V}$ values for the 281 fundamental mode Cepheids in M 31 for different cuts. Error bars indicate the error on the mean. The dashed line represents the adopted distance determination of $(m-M)_{0}=24.32 \pm 0.12 \mathrm{mag}$. Left: each distance determination includes all Cepheids with period longer than the specified value. Right: each distance determination includes all Cepheids with amplitude larger than the specified value.

From the considerations above, the M31 distance modulus obtained from the studied sample of Cepheids is $(m-M)_{0} \simeq$ $\left(V_{0}-M_{V}\right)_{\mathcal{A}>0.8}=24.32 \pm 0.12 \mathrm{mag}$. This value is compatible with most distance determinations found in the literature and the assumed distance modulus in Sect. 5.3. Considering that some large amplitude Cepheids may still be affected by blends, the distance modulus we derive could have a slight negative bias. Note that our final value is 0.09 mag larger than the weighted mean distance modulus for the 281 FM Cepheids $\left(\left(V_{0}-M_{V}\right)=24.23 \pm 0.12 \mathrm{mag}\right)$. Therefore, blending is clearly an important effect that has to be considered when obtaining extragalactic distance determinations. In fact, blending has nearly the same impact on the final distance determination for M 31 as the metallicity correction.

\section{Summary}

As a result of a variability survey in the North-Eastern quadrant of M 31 (Paper I), 416 Cepheids were detected and measured with a large number of epochs $(\sim 250)$ per filter $(B$ and $V)$. The large number of detected Cepheids has allowed the direct study of their period distribution. The results reveal that the period distributions in M31 and the MW are extremely similar and that the Cepheid sample obtained is almost as complete as the DDO MW sample. The only difference may be the lack of long period Cepheids.

The large number of epochs obtained in both filters permits an accurate pulsation mode identification for $240 \mathrm{FM}$ and 75 FO Cepheids. Although some FO Cepheids were previously detected in M31 (Fliri et al. 2006), our sample represents an important increase in the number of detected FO pulsators and opens a new window to study the basic properties of these stars in another Local Group galaxy. The sample of FM Cepheids was completed with 41 long period (i.e., longer than 8 days) Cepheid

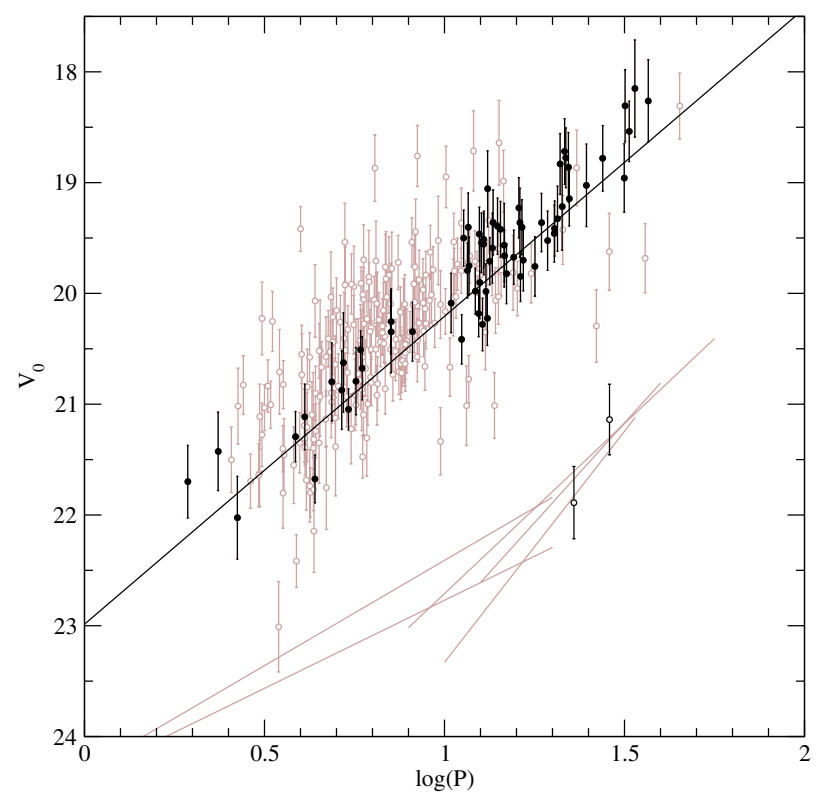

Fig. 9. Absorption-corrected $V$ magnitude as a function of period for 281 fundamental mode Cepheids. Empty grey circles: complete fundamental mode sample. Filled black circles: Cepheids with $\mathcal{A}_{V}>$ 0.8 mag. Empty black circles: two stars with $\mathcal{A}_{V}>0.8$ mag excluded from the distance determination. Black line: Udalski et al. (1999) P-L relationship, for a distance modulus to M 31 of 24.44 (Ribas et al. 2005) and a mean metallicity correction of $-0.1 \mathrm{mag}$ (Sect. 5.2). Grey lines: P-L relationships for type II Cepheids as given by Alcock et al. (1998) at distance of M31.

variables, resulting in $281 \mathrm{FM}$ Cepheids. The posterior analysis revealed that at least three of these stars are type II Cepheids.

The analysis of the P-L relationship for the FM Cepheids reveals a large scatter, which is not explained solely through the effects of interstellar absorption and metallicity. Although additional efforts are needed to reduce the obtained uncertainties, a new method to compute the effect of blending is presented. The exact dependence of the amplitudes among different passbands, as well as more precise amplitude determinations, would greatly improve the results shown here. Even by considering the large uncertainties, the large number of studied Cepheids provides an accurate characterization of the mean blending values. We conclude that the most likely cause of blending seems to be the light from unresolved stars belonging to the same stellar associations or clusters as Cepheid variables.

The effect of blending has been shown to be larger than 0.09 mag in the distance modulus to M 31, thus having an effect as important as the metallicity correction. Therefore, blending should always be taken into account when obtaining extragalactic distance determinations with Cepheids. It has also been shown that an amplitude cut is more adequate for obtaining unbiased distance determinations than a period cut, since the effect of blending is better accounted for. Finally, the large amplitude Cepheids have been used to obtain a distance modulus to M 31 of $(m-M)_{0}=24.32 \pm 0.12 \mathrm{mag}$.

Acknowledgements. The authors are very grateful to L. M. Macri for useful comments during the preliminary stage of this work. Thanks are also due to the anonymous referee for providing valuable comments and information on additional blending determinations. The David Dunlap Observatory and the OGLE teams are also acknowledged for making all their data publicly available in FTP form. This program was supported by the Spanish MCyT grant AyA200615623-C02-01/02. F.V. acknowledges support from the Universitat de Barcelona through a BRD fellowship. I.R. acknowledges support from the Spanish MEC through a Ramón y Cajal fellowship. 


\section{References}

Alcock, C., Allsman, R. A., Alves, D. R., et al. 1998, AJ, 115, 1921 Antonello, E. 2002, A\&A, 391, 795

Antonello, E., Poretti, E., \& Reduzzi, L. 1990, A\&A, 236, 138

Antonello, E., Fugazza, D., \& Mantegazza, L. 2002, A\&A, 388, 477

Baade, W., \& Arp, H. 1964, ApJ, 139, 1027

Baade, W., \& Swope, H. H. 1965, AJ, 70, 212

Beaulieu, J. P., Grison, P., Tobin, W., et al. 1995, A\&A, 303, 137

Becker, S. A., Iben, I., \& Tuggle, R. S. 1977, ApJ, 218, 633

Benedict, G. F., McArthur, B. E., Feast, M. W., et al. 2007, AJ, 133, 1810

Bresolin, F., Pietrzyński, G., Gieren, W., \& Kudritzki, R.-P. 2005, ApJ, 634, 1020

Cotton, W. D., Condon, J. J., \& Arbizzani, E. 1999, ApJS, 125, 409

Feast, M. 1999, PASP, 111, 775

Fernie, J. D., Evans, N. R., Beattie, B., \& Seager, S. 1995, Infor. Bull. Variable Stars, 4148, 1

Fitzpatrick, E. L. 1999, PASP, 111, 63

Fitzpatrick, E. L., Ribas, I., Guinan, E. F., Maloney, F. P., \& Claret, A. 2003, ApJ, 587, 685

Fliri, J., Riffeser, A., Seitz, S., \& Bender, R. 2006, A\&A, 445, 423

Freedman, W. L., Madore, B. F., Gibson, B. K., et al. 2001, ApJ, 553, 47

Gibson, B. K., Maloney, P. R., \& Sakai, S. 2000, ApJ, 530, L5

Gieren, W., Storm, J., Barnes, III, T. G., et al. 2005, ApJ, 627, 224

Hubble, E. P. 1929, ApJ, 69, 103

Landolt, A. U. 1992, AJ, 104, 340

Leavitt, H. S., \& Pickering, E. C. 1912, Harvard College Observatory Circular, 173,1

Macri, L. M. 2004, in Variable Stars in the Local Group, ed. D. W. Kurtz, \& K. R. Pollard, ASP Conf. Ser. 310, IAU Colloq., 193, 33
Macri, L. M., Stanek, K. Z., Bersier, D., Greenhill, L. J., \& Reid, M. J. 2006, ApJ, 652, 1133

Mochejska, B. J., Macri, L. M., Sasselov, D. D., \& Stanek, K. Z. 2000, AJ, 120, 810

Mochejska, B. J., Kaluzny, J., Stanek, K. Z., Sasselov, D. D., \& Szentgyorgyi, A. H. 2001, AJ, 122, 2477

Mochejska, B. J., Macri, L. M., Sasselov, D. D., \& Stanek, K. Z. 2004, in Variable Stars in the Local Group, ed. D. W. Kurtz, \& K. R. Pollard, ASP Conf. Ser. 310, IAU Colloq., 193, 41

Ngeow, C.-C., Kanbur, S. M., Nikolaev, S., Tanvir, N. R., \& Hendry, M. A. 2003, ApJ, 586, 959

Petersen, J. O. 1986, A\&A, 170, 59

Pietrzyński, G., Gieren, W., Udalski, A., et al. 2004, AJ, 128, 2815

Ribas, I., Jordi, C., Vilardell, F., et al. 2005, ApJ, 635, L37

Saha, A., \& Hoessel, J. G. 1990, AJ, 99, 97

Sakai, S., Ferrarese, L., Kennicutt, Jr., R. C., \& Saha, A. 2004, ApJ, 608, 42

Samus, N. N., Durlevich, O. V., et al. 2004, VizieR Online Data Catalog, II/250

Sandage, A., Tammann, G. A., \& Reindl, B. 2004, A\&A, 424, 43

Schwarzenberg-Czerny, A. 1996, ApJ, 460, L107

Simien, F., Pellet, A., Monnet, G., et al. 1978, A\&A, 67, 73

Udalski, A., Szymanski, M., Kubiak, M., et al. 1999, Acta Astron., 49, 201

Udalski, A., Wyrzykowski, L., Pietrzynski, G., et al. 2001, Acta Astron., 51, 221

van Leeuwen, F., Feast, M. W., Whitelock, P. A., \& Laney, C. D. 2007, MNRAS, 379,723

Vilardell, F., Ribas, I., \& Jordi, C. 2006, A\&A, 459, 321

Wozniak, P. R. 2000, Acta Astron., 50, 421

Zakrzewski, B., Ogloza, W., \& Moskalik, P. 2000, Acta Astron., 50, 387

Zaritsky, D., Kennicutt, Jr., R. C., \& Huchra, J. P. 1994, ApJ, 420, 87 